\title{
Field-portable optofluidic plasmonic biosensor for wide-field and label-free monitoring of molecular interactions
}

\author{
Ahmet F. Coskun ${ }^{1,3}$, Arif E. Cetin ${ }^{2,5}$, Betty C. Galarreta, ${ }^{2,4}$, Daniel Adrianzen Alvarez ${ }^{2}$, \\ Hatice Altug ${ }^{2,5, \dagger}$, and Aydogan Ozcan ${ }^{1,6,8}$ \\ ${ }^{1}$ Departments of Electrical Engineering and Bioengineering, University of California, Los Angeles (UCLA), CA 90095, USA \\ ${ }^{2}$ Department of Electrical and Computer Engineering, Boston University, MA 02215, USA \\ ${ }^{3}$ Division of Chemistry and Chemical Engineering, California Institute of Technology, Pasadena, CA, 91125 \\ ${ }^{4}$ Pontificia Universidad Catolica del Peru, Departamento de Ciencias-Quimica, Avenida Universitaria 1801, Lima 32, Peru \\ ${ }_{5}^{5}$ Bioengineering Department, Ecole Polytechnique Federale de Lausanne (EPFL), Lausanne CH-1015 Switzerland \\ ${ }^{6}$ California NanoSystems Institute (CNSI), University of California, Los Angeles (UCLA), CA 90095, USA \\ §ozcan@ucla.edu, †hatice.altug@epfl.ch
}

\begin{abstract}
We demonstrate a field-portable optofluidic plasmonic sensing device, weighing $40 \mathrm{~g}$ and $7.5 \mathrm{~cm}$ in height, which merges plasmonic microarrays with dual-wavelength lensfree on-chip imaging for real-time monitoring of protein binding kinetics.
\end{abstract}

Label-free biodetection methods have great potential for early screening of health conditions in point-of-care settings. There is a growing interest in the development of high-throughput and field deployable biosensors that can simultaneously measure multiple disease markers without the need for any labeling and sophisticated sample preparation protocols. Motivated by these important needs, we have demonstrated a multiplexed optofluidic plasmonic biosensing device that can monitor molecular binding events in real-time over a wide field-of-view that is larger than $20 \mathrm{~mm}^{2}[1]$.

This hand-held biodetection platform, shown in Figure 1a and 1b, combines microfluidics enabled plasmonic microarrays with dual-wavelength lensfree on-chip imaging. This compact device, weighing $40 \mathrm{~g}$ and $7.5 \mathrm{~cm}$ in height, comprises two distinct light emitting diodes (LEDs) which have peak wavelengths at $820 \mathrm{~nm}$ and $880 \mathrm{~nm}$, i.e., adjusted to the left and right sides of the plasmonic mode that is indicated with a black curve in Figure 1c; this configuration generates uniform light illumination over our plasmonic chip, which is composed of subwavelength nanohole arrays. The transmission of this dual-LED illumination through the plasmonic chip is then detected by a Complementary Metal-Oxide-Semiconductor (CMOS) imager chip, which samples two diffraction patterns for each plasmonic sensor at the detector array. The change in the refractive index of the medium causes a spectral shift in the plasmonic mode (see Figure 1c for the spectral shift from $852 \mathrm{~nm}$ to $878 \mathrm{~nm}$, as denoted with a green curve), such that the signal of the diffraction pattern corresponding to the left LED decreases while that of the right LED increases (see Figure 1d). We then perform quantitative analysis of molecular binding events through the use of a ratiometric analysis that is performed on these acquired dual diffraction patterns [1].

This biosensing approach has several important features: $(i)$ the use of plasmonic nanohole arrays provides high sensitivity for biomolecule quantification at the active sensing region, (ii) the use of lensfree on-chip imaging provides a multiplexing capability of biodetection by simultaneously monitoring thousands of plasmonic nanostructures, and (iii) the use of microfluidics enables automated sample preparation and real-time monitoring of biomolecular interactions over a wide dynamic range, all on the same platform.
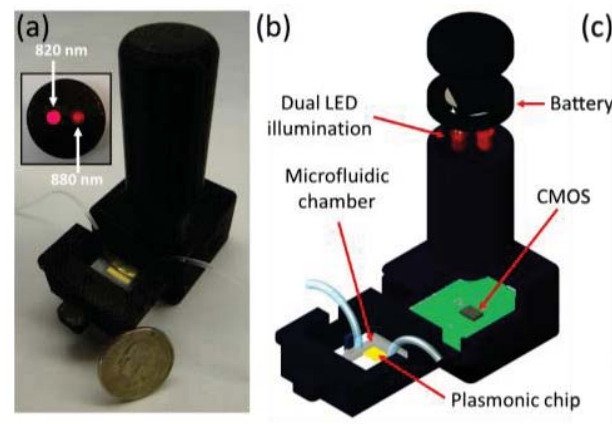

(c)
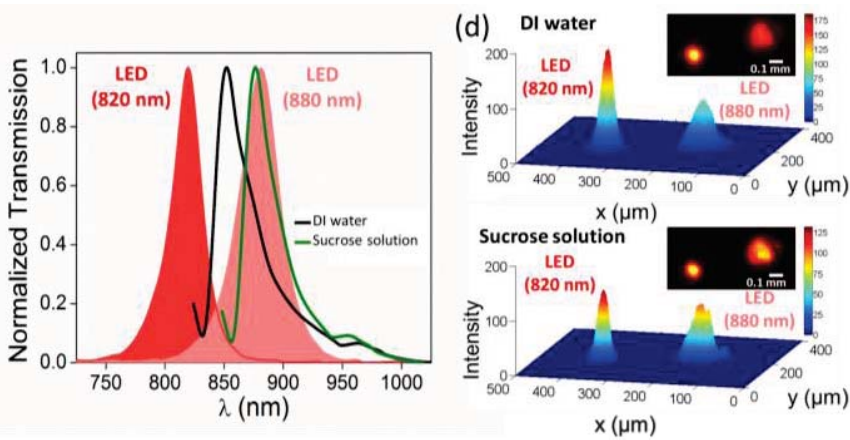

Figure 1. (a) Picture and (b) schematic illustration of the optofluidic lensfree plasmonic biosensing device. (c) Solid curves: Spectral shift of the plasmonic mode (black curve) under a higher refractive index medium (green curve). Filled curves: Spectra of two different color LEDs. (d) Changes in the intensity of the dual color diffraction patterns of nanohole arrays embedded in DI-water and sucrose solution. 
In order to create affordable wide-area sensor chips for field applications, we also introduced a high-throughput fabrication technique utilizing deep ultra-violet (UV) lithography that can create thousands of plasmonic nanostructures at low-cost. From a 4-inch wafer, we simultaneously fabricated more than 50 plasmonic sensing devices using deep UV lithography, which dramatically improves the throughput of patterning compared to classical fabrication methods, e.g., based on electron-beam lithography.

For sensitivity analyses, we used sugar solutions with various concentrations, ranging from 0.055 to $1.1 \mathrm{~mol} / \mathrm{L}$ (Figure 2a). Here, the wavelength shift (Figure 2b) detected by an optical spectrometer and the intensity ratio change (Figure 2c) calculated from the lensfree images demonstrate a similar behavior/trend for increasing sucrose concentrations. Our experiments resulted in a detection limit (denoted with red lines in Figures $2 \mathrm{~b}$ and $2 \mathrm{c}$ ) of: (i) 0.002 RIU (refractive index unit) based on the optical spectrometer data, and (ii) 0.001 RIU using the lensfree imaging data. Compared to our previous handheld design utilizing a single LED configuration [2], the presented optofluidic biosensor achieves more than two fold improvement in the detection sensitivity owing to the ratiometric color analysis performed on the lensfree images.
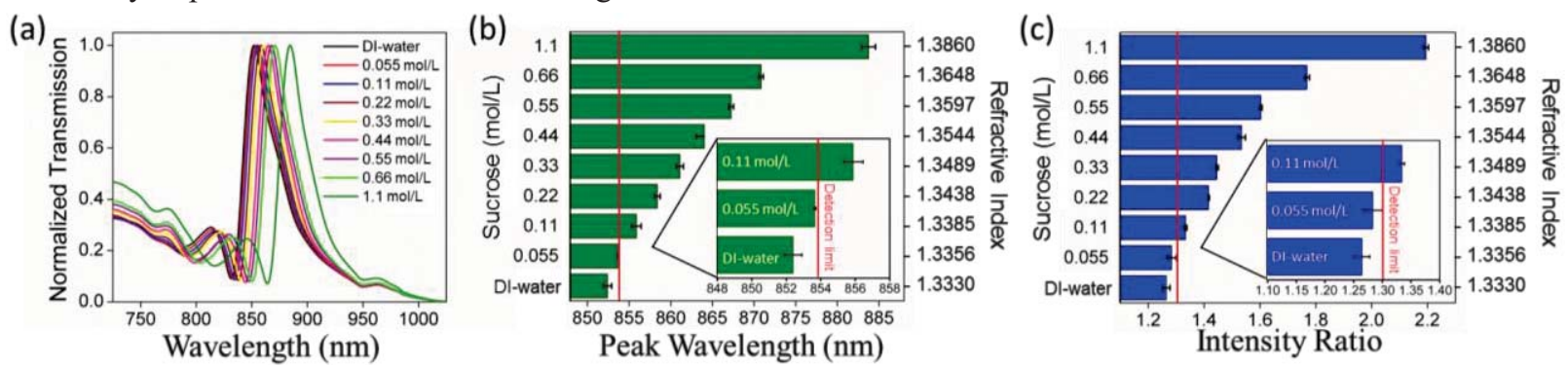

Figure 2. (a) Spectral characteristics of the plasmonic sensor embedded in several dilutions of sucrose samples. (b) Peak wavelength shift in the plasmonic mode measured by an optical spectrometer. (c) Corresponding intensity ratios calculated from dual-color diffraction patterns sampled on a lensfree imager. The detection limit is indicated with a red line in both cases.

Utilizing a microfluidic chamber, our biosensor can quantitatively analyze the interactions of protein complexes. We performed time-lapse experiments in order to monitor the association phase of protein $\operatorname{IgG}(200 \mu \mathrm{g} / \mathrm{mL})$ onto protein $\mathrm{A} / \mathrm{G}(100 \mu \mathrm{g} / \mathrm{mL})$, as schematically illustrated in Figure 3a. This real-time binding process alters the local refractive index in such a way that the optical response of the plasmonic substrate exponentially varies in time. Figure $3 \mathrm{~b}$ shows the spectral shifts in the plasmonic response measured by an optical spectrum analyzer (obtained from a single sensor) and Figure $3 \mathrm{c}$ shows the relative intensity ratio computed from the dual-wavelength lensfree sensor (obtained from 3 individual sensors). As shown in Figure 3c-inset, binding of protein IgG onto protein $\mathrm{A} / \mathrm{G}$ over 80 minutes yields $\sim 6 \mathrm{~nm}$ shift in the peak wavelength. Based on our analytical model for the association phase, we can fit an exponential to both of our data sets as follows:

$$
\Delta \lambda=6.448 \times\left(1-\mathrm{e}^{-0.0005333 \times \mathrm{t}}\right) \text { and } \mathrm{IR}=0.10188 \times\left(1 \mathrm{e}^{-0.0005685 \times \mathrm{t}}\right)
$$

where $\Delta \lambda$ is the spectral shift from the initial peak wavelength position at time $t$ and $I R$ is the intensity ratio change from the initial intensity ratio at time $t$. Equation 1 shows that the two measurements (spectrum analyzer vs. lensfree imaging) provided comparable exponential coefficients, 0.0005333 (spectrometer) and 0.0005685 (lensfree), demonstrating that our lensfree biosensor can be used for real-time analysis of protein-protein binding events.
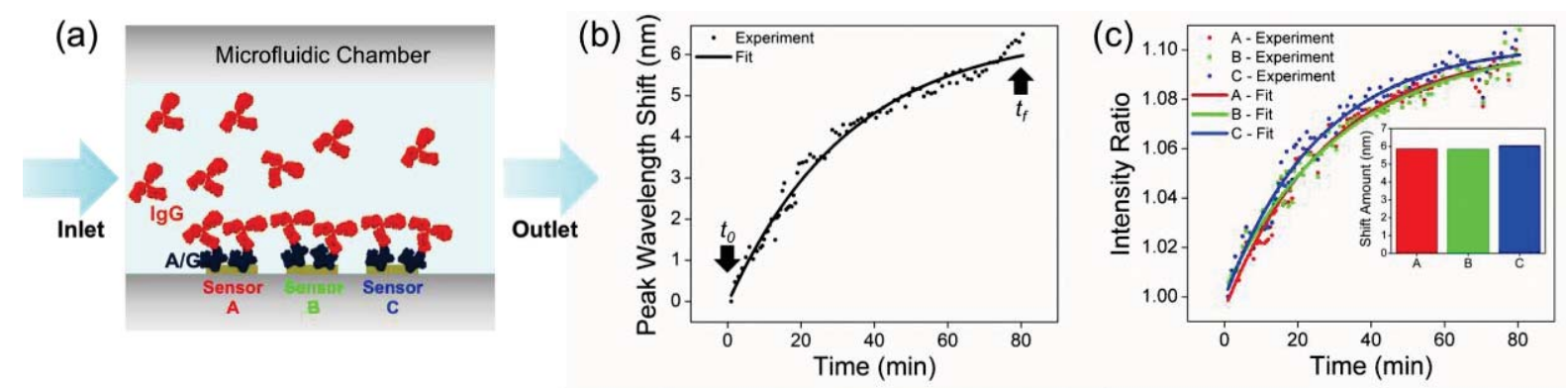

Figure 3. (a) Schematics of protein $\mathrm{A} / \mathrm{G}-\mathrm{IgG}$ binding events on 3 different plasmonic sensors within a microfluidic chamber. (b) Real-time experimental data and exponential fit for the spectral shift in the plasmonic mode measured by a spectrometer. (c) Experimental data and exponential fit for the intensity ratio obtained by the lensfree imager. Figure inset shows the amount spectral variation after the injection of protein $\mathrm{IgG}$, demonstrating a total shift of $\sim 6 \mathrm{~nm}$ over 80 minutes.

[1] Ahmet F. Coskun, Arif E. Cetin, Betty C. Galarreta, Daniel Adrianzen Alvarez, Hatice Altug, and Aydogan Ozcan, Scientific Reports, 4, 6789 (2014).

[2] Arif E. Cetin, Ahmet F. Coskun, Betty C. Galarreta, Min Huang, David Herman, Aydogan Ozcan and Hatice Altug, Light: Science \& Applications, 3, e122 (2014). 